\title{
FRET-based method for evaluation of the efficiency of reversible and irreversible sonoporation
}

\author{
Paulius Ruzgys \\ Mindaugas Tamošiūnas \\ Vanesa Lukinsone \\ Saulius Šatkauskas
}




\title{
FRET-based method for evaluation of the efficiency of reversible and irreversible sonoporation
}

\author{
Paulius Ruzgys, ${ }^{a}$ Mindaugas Tamošiūnas, ${ }^{a}$ Vanesa Lukinsone, ${ }^{b}$ and Saulius Šatkauskas ${ }^{a, \star}$ \\ aVytautas Magnus University, Biophysical Research Group, Faculty of Natural Sciences, Kaunas, Lithuania \\ bUniversity of Latvia, Institute of Atomic Physics and Spectroscopy, Riga, Latvia
}

\begin{abstract}
It is widely known that not all of the treated cells survive after introduction of exogenous molecules via any physical method. Therefore, it is important to develop methods that would allow simultaneous evaluation of both molecular delivery efficiency and cell viability. This study presents Förster resonance energy transfer (FRET)-based method that allows molecular transfer and cell viability evaluation in a single measurement by employing two common fluorescent dyes, namely, ethidium bromide and trypan blue. The method has been validated using cell sonoporation. The FRET-based method allows the efficiency evaluation of both reversible and irreversible sonoporation in a single experiment. Therefore, this method could be used to reduce time, labor, and material cost while improving the accuracy of evaluations. ๑2017 Society of Photo-Optical Instrumentation Engineers (SPIE) [DOI: 10.1117/1.JBO.22.9.097001]
\end{abstract}

Keywords: Förster resonance energy transfer; trypan blue; ethidium bromide; cell viability; sonoporation; molecule sonotransfer. Paper 170180R received Mar. 30, 2017; accepted for publication Aug. 21, 2017; published online Sep. 14, 2017.

\section{Introduction}

Fast and reliable cell viability assays are highly desired for any experiments with cells in vitro. For example, cell viability evaluation is important when physical methods based on temporary increase in membrane permeability, such as sonoporation, are used to deliver exogenous molecules to the cells. Cell sonoporation can be induced by exposing cells to high intensity ultrasound (US) in the presence of microbubbles (MB). This leads to facilitated delivery of drug, gene, or other bioactive molecules across sonoporated cell membrane. ${ }^{1}$ However, sonoporation can also induce the death of the treated cells. ${ }^{2-4}$ Therefore, it is crucial to evaluate cell viability when performing sonotransfer experiments. A few membrane integrity-based cell viability assays are compatible with sonoporation experiments; these use propidium iodide, ${ }^{1,5,6}$ ethidium bromide (EB),${ }^{7,8}$ or trypan blue (TB $)^{7-9}$ fluorescent dyes. It should be noted that these fluorescent dyes have to be added to the cell suspension 10 to $20 \mathrm{~min}$ after the sonoporation treatment for estimation of cell viability.

Cell viability assays based on cell metabolic activity evaluation, e.g., MTT or XTT assays, ${ }^{10,11}$ can also be employed for cell viability evaluation after sonoporation. However, as previously indicated, metabolic activity-based assays may lead to imprecise evaluation of cell viability. ${ }^{12}$ Another conventional cell viability test is clonogenic assay, which is accurate but time and labor consuming. ${ }^{13,14}$

It should be noted that none of the presented viability assays enable simultaneous evaluation of cell viability and efficiency of exogenous molecule transfer. Here, we present a simple method for the evaluation of sonoporation efficiency and cell viability in a single experiment that utilizes commonly used fluorescent dyes: EB and TB. We also show that interaction of both fluorescent dyes used in this method is at least partially related to Förster resonance energy transfer (FRET). EB emission energy

*Address all correspondence to: Saulius Šatkauskas, E-mail: saulius .satkauskas@vdu.It is absorbed by TB through radiationless energy transfer in a protein-rich environment, such as the cytoplasm of the cell. Understanding the mechanisms of the method can help to optimize the conditions to get more reliable results not only after cell sonoporation but also after other physical cell treatment such as cell electroporation. At this point, we show that the assay is compatible not only with fluorescence microscopy but also with flow cytometry (FC) that eventually reduce assay cost and time for assay performance.

\section{Methods}

\subsection{US Hardware Setup}

One-megahertz unfocused US transducer, having $19 \mathrm{~mm}$ diameter (UAB Medelkom, Vilnius, Lithuania), was used. The transducer was calibrated using using polyvinylidene flouride needle needle hydrophone $(0.2 \mathrm{~mm}$ diameter, Precision Acoustics, Dorchester, United Kingdom). The transducer was connected to the US generation system (Picotech LTD, Cambridgeshire, United Kingdom), which delivered a US signal of 2-s duration and 500-kPa peak negative pressure (PNP), with $10 \%$ duty cycle at pulse repetition frequency of $1000 \mathrm{~Hz}$. Polystyrene cuvette with $10 \times 10 \mathrm{~mm}$ internal dimensions (Carl Roth $\mathrm{GmbH}+\mathrm{Co}$, Germany) was placed in the center of the transducers acoustic field and kept in distilled deionized water tank maintained at room temperature.

\subsection{Suspension Absorbance and Emission Measurements}

Beckman Coulter DU 800 spectrophotometer (Conquer Scientific, San Diego) was used to measure the absorption spectra in the range between 200 and $800 \mathrm{~nm}$ wavelengths. Quartz cuvettes (110-QS light path $1 \mathrm{~mm}$ Hellma Analytics,

$1083-3668 / 2017 / \$ 25.00$ @ 2017 SPIE 
Mullheim Germany) were used during suspension absorption measurements.

EB and TB steady-state fluorescence emission was recorded using a fiber optic spectrophotometer (AvaSpec ULS2048L, Avantes, The Netherlands). Diode-pumped solid-state laser light (473 nm, CW, maximum output $100 \mathrm{~mW}$, Altechna, Lithuania) was collimated to the central fiber of the Y-shaped bundle (FCUV400-2-SR, Avantes, The Netherlands) for EB or TB fluorescence excitation. Six circularly positioned fibers of the bundle were used to collect the fluorophore emission signal, and the long pass filter $\left(T_{516-800 \mathrm{~nm}}>60 \%\right)$ was inserted before the spectrophotometer to suppress the transmittance of the excitation light. Polystyrene cuvettes (Carl Roth $\mathrm{GmbH}+\mathrm{Co}$, Germany) were used for all of the emission measurements.

Time correlated single photon counting (TCSPC) setup was described in detail previously. ${ }^{15}$ The single-spot measurement setup was comprised of a laser controller and a picosecond/CW laser: $470 \mathrm{~nm}$, pulse half-width $73 \mathrm{ps,} \mathrm{repetition} \mathrm{rate} 20 \mathrm{MHz}$ mod. (LDH-D-C-470, PicoQuant, Berlin, Germany) with $200-\mu \mathrm{m}$ silica core optical fiber output via the SMA-connector. The excitation fiber of the Y-shaped bundle was directed to the sample cuvette and six additional fibers were used to deliver the fluorescence light via a monochromator to the photon counting detector (PMC-100-4 Becker\&Hickl, Berlin, Germany). Data were collected at $626 \mathrm{~nm}$ emission wavelength. In order to evaluate the emission decay, totally 45,000 to 350,000 photons were collected; the collection time of the signal was $4 \mathrm{~s}$. The laser controller and the photon counting detector were connected to a PC with a data-processing card (SPC-150 TCSPC module, Becker\&Hickl, Berlin, Germany). SPCImage 5.0 was used for data analysis.

\subsection{Fluorescence Microscopy and Processing with ImageJ}

Bright field and fluorescence microscopy images of the cells were taken using Motic Pro 285 B camera, mounted on an inverted fluorescence microscope Motic AE-31 (Xiamen, China). EB was excited with $560 \pm 40 \mathrm{~nm}$ wavelength light. Emission was measured at $630 \pm 60 \mathrm{~nm}$ wavelengths.

Images were processed with the open source imaging program, ImageJ. Fluorescence visibility was enhanced, using a rolling ball algorithm for background subtraction. ${ }^{16}$ The average cell radii in taken images were $9.54 \pm 0.44$ pixels. Therefore, 10-pixel radius for the rolling ball was selected to make correct conversion with the lowest noise possible. After background subtraction, image grayscale dilation was applied by replacing each pixel in the image with the largest pixel value in 2 pixel range to remove background noise introduced after conversion. This also determined the detection levels for cell fluorescence area representing $4 \times 4$ pixels. A processed fluorescence image in combination with the image of the same cells under brightfield illumination enabled visualization of unaffected, sonoporated, and killed cells.

\subsection{Flow Cytometry Measurements}

Flow cytometer BD Accuri C6 (BD Biosciences, San Jose) was used for the FC experiments. All cell suspensions were excited with $488 \mathrm{~nm}$ laser. To avoid debris, flow cytometer was set on fast flow rate $(66 \mu \mathrm{L} / \mathrm{min})$. Cells were gated on graph with forward scattering and side scattering axes, and 10,000 events in the gated area were measured. Unaffected, reversibly sonoporated, and killed cells were separated into groups by their measured emission in FL2 $(585 \pm 40 \mathrm{~nm})$ spectrum.

\subsection{Chemicals}

Sonovue MB's were purchased from Bracco (Bracco Research, Geneva, Switzerland) and prepared by diluting the powder in a sterile 5-mL $0.9 \% \mathrm{NaCl}$ solution. $\mathrm{EB}, \mathrm{TB}$, and all of the cell culture reagents were purchased from Sigma (Sigma-Aldrich, St. Louis, Missouri). For FC experiments, TB solution was filtered through $0.22-\mu \mathrm{m}$ pore diameter filter.

\subsection{Cell Culture Maintenance and Experimental Procedure}

Chinese hamster ovary $(\mathrm{CHO})$ cell culture was used as an object for sonotransfer. Cells were cultured in Dulbecco's Modified Eagle Medium supplemented with $10 \%$ fetal bovine serum (FBS), $1 \%$ penicillin-streptomycin, and $1 \%$ L-glutamine (Sigma-Aldrich, St. Louis, Missouri). Cells were incubated at $37^{\circ} \mathrm{C}$ in a humidified atmosphere with a $5 \% \mathrm{CO}_{2}$ concentration (NU-2500E, NuAire, Plymouth, Minnesota). Cell culture was routinely subcultured every 3 or 4 days.

For the sonoporation experiments, the sonoporation cuvette was filled to $1 \mathrm{~mL}$ volume, containing $3.3 \times 10^{5}$ cells $/ \mathrm{mL}$, $1.32 \times 10^{7} \mathrm{MB} / \mathrm{mL}$, and $600 \mu \mathrm{M}$ EB final concentration. The latter results in 40 to $1 \mathrm{MB}$ to cell ratio. After US treatment, cell suspension was incubated for $10 \mathrm{~min}$. After that, cells were centrifuged at $200 \mathrm{~g}$ for $2 \mathrm{~min}$. Supernatant was removed and the pellet was suspended with $20 \mu \mathrm{L}$ (for fluorescence microscopy measurements) or $200 \mu \mathrm{L}$ (for flow cytometer measurements) of phosphate-buffered saline. Afterward, $20 \mu \mathrm{L}$ of TB $(0.4 \%)$ (for fluorescence microscopy measurements) or $10 \mu \mathrm{L}$ of TB $(0.4 \%)$ (for FC measurements) was applied to cell suspension. After 1min incubation, images of the cells in the same field were taken using bright field and fluorescence microscopy or FC measurements were made. Duration between the addition of TB and the measurements never exceeded $5 \mathrm{~min}$.

\section{Results}

The aim of this study was to develop a simple, fast, and cheap method that would allow distinguishing between unaffected, reversibly sonoporated (sonotransfered with EB), and irreversibly sonoporated (dead) cells.

First, we examined the coincidence between the EB fluorescence spectrum and the TB absorption spectrum [Fig. 1(a)]. Presented overlapping spectra indicate the possibility of TB to quench EB fluorescence via one of two mechanisms: (i) radiative energy transfer when EB fluorescence is absorbed by TB and (ii) radiationless energy transfer from EB to TB (FRET). We investigated $\mathrm{EB}$, the absorption and emission of both fluorophores, as well as quenching by TB in the presence and in the absence of FBS. FBS contains proteins, thus imitating protein-rich environment, similar to cell cytoplasm. In FBS environment, broad emission spectrum of EB centered at $620 \mathrm{~nm}$ [Fig. 1(a), dashed line]. TB absorption spectrum in FBS had maximum at $595 \mathrm{~nm}$ [Fig. 1(a), dashed line], whereas in water environment the maximum of TB absorption spectrum was shifted to $583 \mathrm{~nm}$ (data not shown). After mixing both dyes in FBS, EB emission was effectively quenched; it diminished from around 25,000 a.u. (no TB present) to around 4000 a.u. [Fig. 1(b), dotted line]. 

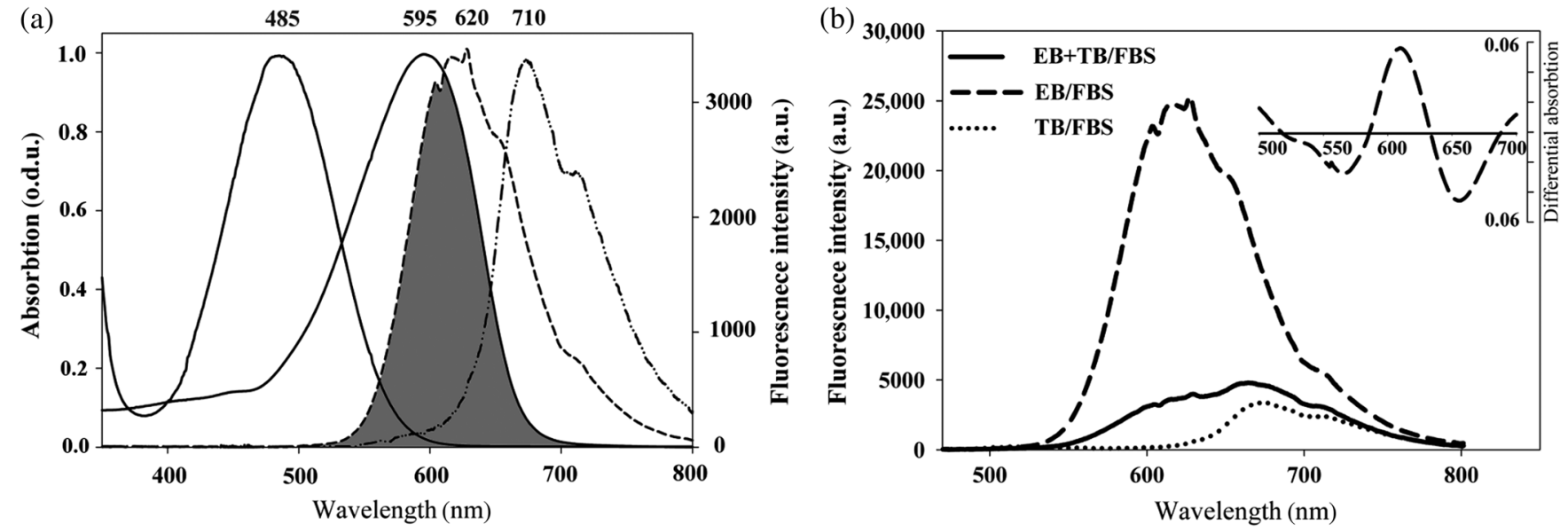

Fig. 1 (a) Spectral overlap between EB emission and TB absorption (filled gray area). Normalized spectra of EB absorption (470 nm peak, solid line), TB absorption (595 nm peak, solid line), EB emission (620 nm peak, dashed line), and TB emission (710 nm peak, dotted line) in FBS. (b) Emission spectra of EB (dashed line), TB (dotted line), and EB in the presence of TB (solid line) in FBS. Insert: absorption difference of TB between FBS and water.

To understand the influence of the observed TB absorption spectrum shift in FBS environment on the quenching of EB, additional data analysis was performed. Figure 1(b) (insert) shows the increase of differential TB absorption in 500 to $700 \mathrm{~nm}$ spectral region when TB was diluted in FBS. Notably, EB emission maximum is positioned between these values. Therefore, the slight increase in EB emission when in FBS environment $(25,000$ a.u.) as compared to pure water environment (18,000 a.u.) (data not shown) was in part compensated with the increase of TB absorption. Moreover, the significant absorption increase of TB diluted in FBS precisely overlaps with the EB peak in FC filter transmission zone (545 to $625 \mathrm{~nm}$ ), leading to the decrease of donor fluorophore fluorescence.

These findings were utilized to measure EB sonotransfer and cell membrane integrity. Obtained results allowed discrimination among unaffected cells, EB positive cells (reversible sonoporation), and dead cells (irreversible sonoporation). Indeed, experiments with $\mathrm{CHO}$ cell culture showed the capability to discriminate among all three cell groups (see Fig. 2). Images were acquired using fluorescence microscope and processed with the open source imaging program, ImageJ. The bright-field illumination images and the fluorescence images were captured separately and merged together. In Fig. 2(a), red fluorescent cells depict both reversibly sonoporated and dead cell populations, since EB enters both of the cell groups. To discriminate among these groups, TB (which only enters dead cells) was added 15 min after US exposure [Fig. 2(b)]. Cell fluorescence images show that EB emission was quenched by TB only in some of the cells, which allowed the assumption that the cells that were still fluorescent after addition of TB did not have compromised membrane, thus denying TB entry. Moreover, when observed under bright-field illumination, TB intensively stained the dead cells. Therefore, the analysis of merged fluorescence and bright-field illumination images allowed clear separation of the populations of the dead and the viable cells. We tested this new method for evaluation of sonotransfer and cell viability after cell sonoporation experiments, using US conditions described above (1-MHz central frequency, 500-kPa PNP,

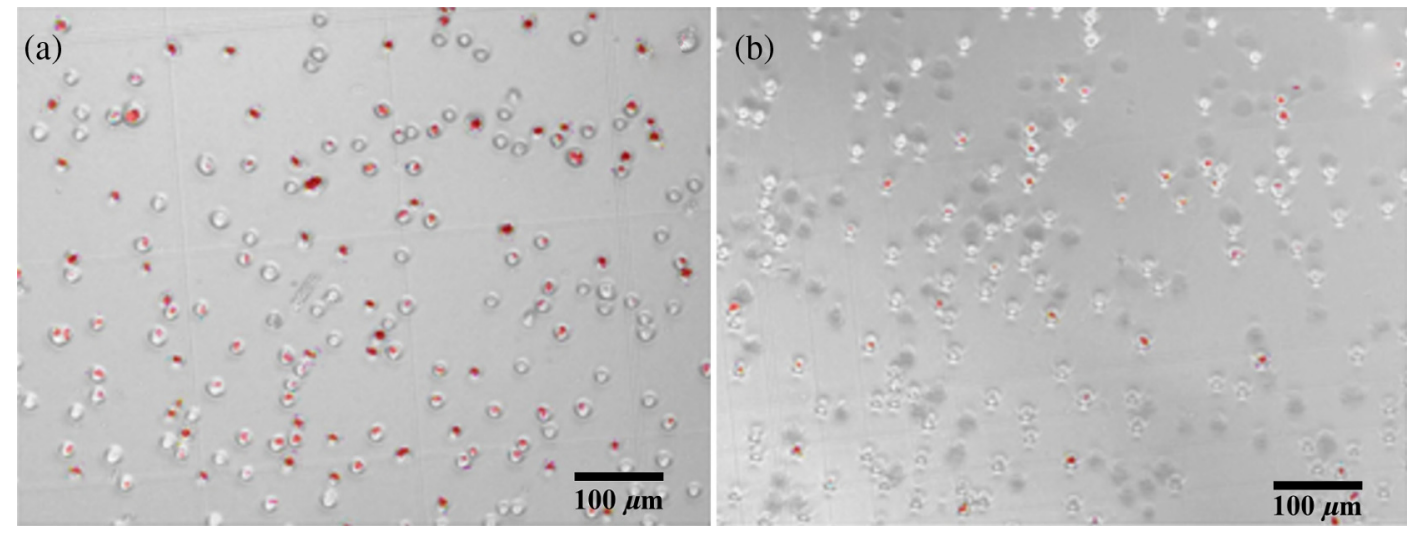

Fig. 2 Microscopy images after sonoporation, (a) in the presence of EB $(600 \mu \mathrm{M})$ alone or (b) EB $(600 \mu \mathrm{M})+\mathrm{TB}(229 \mu \mathrm{M}$ or $0.02 \% \mathrm{w} / \mathrm{v})$. The images were acquired under bright-field illumination or under fluorescence excitation $(560 \pm 40 \mathrm{~nm})$ with emission filter $(630 \pm 60 \mathrm{~nm})$, then merged together after background subtraction using open source program Image J. Cells were insonicated at $1 \mathrm{MHz}$, $500 \mathrm{kPa}$ PNP acoustic pressure, $10 \%$ duty cycle, and $1 \mathrm{kHz}$ repetition frequency for $2 \mathrm{~s}$ in the presence of Sonovue MBs. 

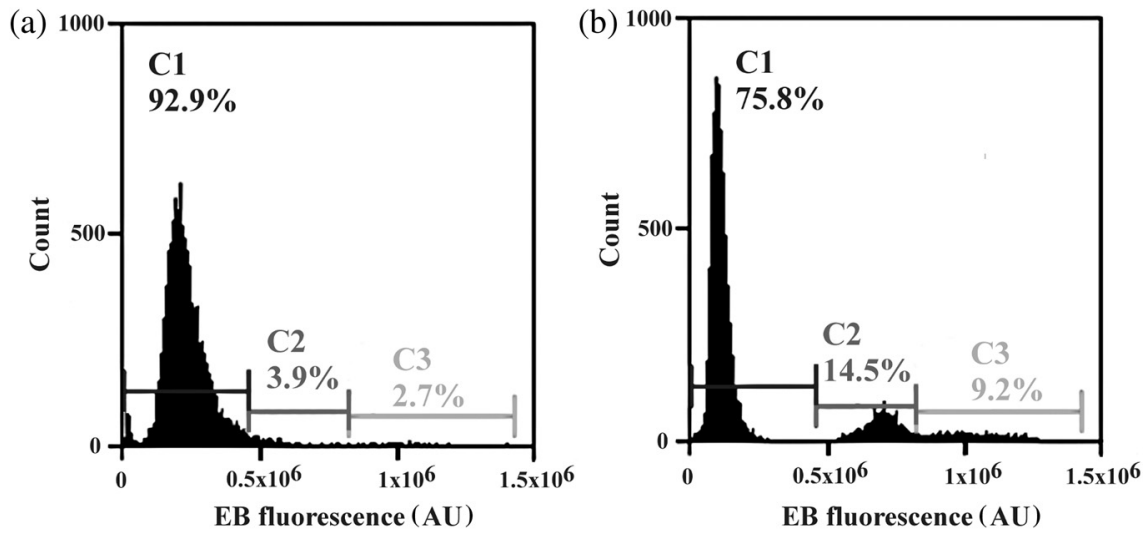

Fig. $3 \mathrm{FC}$ results in the presence of $\mathrm{EB}(600 \mu \mathrm{M})$ and TB $(0.02 \% \mathrm{w} / \mathrm{v})$ at (a) control conditions and (b) after sonoporation. $488 \mathrm{~nm}$ excitation laser and $\mathrm{u}$ emission filter transmitting from 545 to $625 \mathrm{~nm}$ were used in FC. Cells were insonicated at $1 \mathrm{MHz}, 500 \mathrm{kPa}$ PNP acoustic pressure, $10 \%$ duty cycle, and $1 \mathrm{kHz}$ repetition frequency for $2 \mathrm{~s}$ in the presence of Sonovue MBs. C1 represents viable nonaffected cells, C2 represents irreversibly sonoporated (dead) cells, and C3 represents reversibly sonoporated cells.

$10 \%$ duty cycle with pulse repetition frequency of $1000 \mathrm{~Hz}$, and total exposure duration $2 \mathrm{~s}$ ). Cell exposure to US under these conditions resulted in $9.6 \pm 1 \% \mathrm{~EB}$ sonotransfer and $17.3 \pm 0.2 \%$ of dead cells. The rest of the cells were unaffected. In comparison, $6.4 \pm 1.7 \%$ dead and $1.4 \pm 1.4 \%$ EB positive cells were observed in control.

The same experiment was performed to test the compatibility of the new sonotransfer efficiency and cell viability evaluation method with FC. Figure 3 shows FC results in control group [Fig. 3(a)] and after US application [Fig. 3(b)]. In the control group, only one cell population peak (C1) with low autofluorescence intensity was detected [Fig. 3(a)]. Therefore, $\mathrm{C} 1$ region corresponded to viable and unaffected cell population. When cells were treated with US, three populations were distinguishable [Fig. 3(b)]. The low fluorescent cell population in $\mathrm{C} 1$ region denoted unaffected cells with no EB inside. The population with the highest fluorescence (marked as C3 region) showed the cells with EB fluorescence that was not quenched by TB, thus indicating that no TB entered the cells. The last cell population, which is more fluorescent than $\mathrm{C} 1$, but less fluorescent than $\mathrm{C} 3$, was marked within $\mathrm{C} 2$ region. This indicated that $\mathrm{C} 2$ population had accumulated EB, but its fluorescence was quenched by the presence of TB inside the cells. Therefore, $\mathrm{C} 2$ population represented dead cells. Both the sonotransfer and the decrease in cell viability shown in Fig. 3 go in agreement with our previous study. ${ }^{3}$

Fluorescence lifetime measurements were employed to investigate the energy transfer pathways between EB and TB. As previously indicated, the experiments in vitro were performed in the absence or in the presence of FBS. The estimation shows that without FBS EB relaxes monoexponentially with the time constant $\tau=1.75 \pm 0.02 \mathrm{~ns}$ (data not shown). The time dependence of EB fluorescence intensity decay measurements is shown in Fig. 4. The bimodal behavior of EB fluorescence decay was observed in the presence of FBS, with the time constants $\tau_{1 \mathrm{~d}}=1.79 \pm 0.1 \mathrm{~ns}$ (short component) and $\tau_{2 \mathrm{~d}}=$ $5.2 \pm 0.14 \mathrm{~ns}$ (long component), Fig. 4(a). A different time dependence of EB relaxation was detected in the presence of FBS and TB. The two-exponent fitting gives slightly different time constants of $\tau_{1 \mathrm{da}}=1.55 \pm 0.08 \mathrm{~ns}$ (short component) and $\tau_{2 \mathrm{da}}=4.67 \pm 0.21 \mathrm{~ns}$ (long component) [Fig. 4(b)].

The main difference between $\mathrm{EtBr}$ fluorescence lifetime in $+\mathrm{TB}$ and $-\mathrm{TB}$ solutions is the statistically significant reduction of both short $\tau_{1}$ and long $\tau_{2}$ components. The mean amplitude
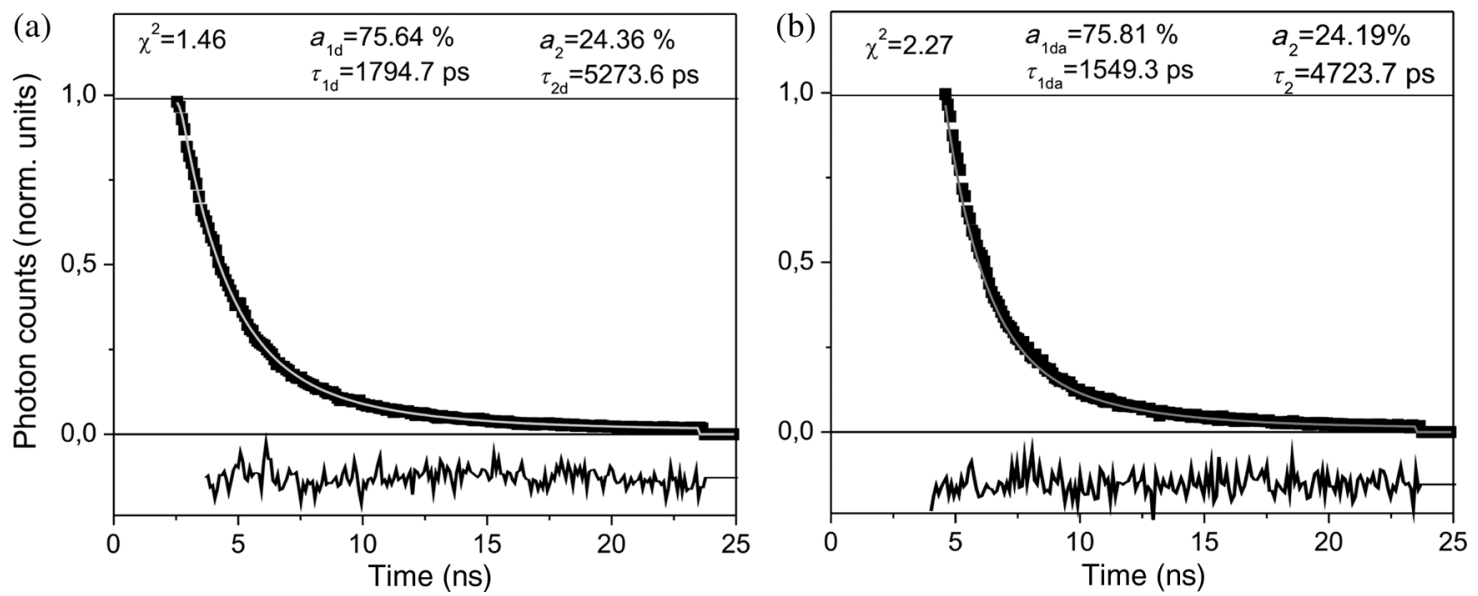

Fig. 4 Kinetic measurements of EB fluorescence in FBS, (a) in the presence and (b) in the absence of TB. EB and FBS final concentration was kept at 6:1 molar ratio. TB:FBS molar ratio was 2.29:1. 
weighted lifetime $\langle\tau\rangle=\sum a_{i} \tau_{i}$ of EtBr was calculated both in the presence $\left(\left\langle\tau_{\mathrm{da}}\right\rangle=2.29 \mathrm{~ns}\right)$ and absence $\left(\left\langle\tau_{\mathrm{d}}\right\rangle=2.61 \mathrm{~ns}\right)$ of the acceptor (TB). FRET efficiency $(E)$ was calculated by $E=1-\left\langle\tau_{\mathrm{da}}\right\rangle /\left\langle\tau_{\mathrm{d}}\right\rangle=12 \%$. These findings allow the conclusion that the loss of EB fluorescence in FBS solution in the presence of TB is mainly governed by FRET.

FRET is expected in the regions where EtBr and TB are colocalized on the protein.

\section{Discussion}

In this work, we present an FRET-based method to evaluate efficiency of reversible and irreversible sonoporation in a single experiment. To this end, we use two common fluorescent dyes: EB and TB, which are already used for cell staining in sonoporation setting. ${ }^{7,8} \mathrm{~TB}$ is also used for animal cell ${ }^{9,17}$ and plant cell staining. ${ }^{18}$ The presented method is based on the EB fluorescence quenching by TB. EB is a DNA-intercalating agent, commonly used as a fluorescent dye. After binding to DNA, the fluorescence of this dye is intensified 10 -fold. ${ }^{19}$ It has also been shown that EB binds to proteins. ${ }^{20} \mathrm{~EB}$ has been already shown to be quenched by a number of compounds, including porphyrin cations and quaternary ammonium compounds, ${ }^{21}$ antitumor drug amsacrine, ${ }^{22}$ natural compound emodin, ${ }^{23}$ or by newly synthetized dibipyridyl ruthenium(II) complex of p-tert-butyltetrathiacalix4arene, ${ }^{24}$ and bromodeoxyuridine. ${ }^{25,26}$ On the other hand, it has also been shown that TB fluorophore could be used as a quenching agent. With its maximal absorption spectrum at pure water being $590 \mathrm{~nm},{ }^{27} \mathrm{~TB}$ has been used to quench cell autofluorescence in retinal pigment epithelia cells, ${ }^{28}$ alveolar macrophages from smokers, ${ }^{29}$ human mesenteric blood vessels, ${ }^{30}$ and extracellular fluorescence. ${ }^{31}$

Our experiments indicate that EB emission can be efficiently quenched by TB absorption in a cellular environment. After sonoporation, a donor fluorophore (in our case EB) has been able to localize in the close proximity to TB, leading to the energy transfer to TB through nonradiative dipole-dipole coupling. ${ }^{32}$ The increase of $\mathrm{EB}$ emission spectrum peak in the FBS environment could be explained by the fact that EB is able to interact with proteins and DNA. ${ }^{20} \mathrm{~TB}$ is also able to interact with proteins, which could explain the shift in absorption peak. ${ }^{33}$ We propose that FRET between EB and TB might be enabled by protein interaction with both of the fluorescent molecules, thus explaining the occurrence of the phenomenon in protein-rich (e.g., intracellular) but not aqueous environment.

EB emission and TB absorption spectra in protein environment strongly coincide with the transmittance characteristics of the standard FC filter. Therefore, the use of both fluorophores can be easily adapted to FC measurements for the detection of sonotransfer efficiency and cell viability. Consequently, this allows discrimination of three distinct cell populations: (i) nonaffected cells, (ii) reversibly sonoporated cells, and (iii) irreversibly sonoporated (dead) cells.

The method can be also adapted for fluorescence microscopy. Indeed, TB both stains the dead cells under visible light and quenches EB fluorescence. For better discrimination of distinct cell populations, microscope images have to be processed with imaging processing software, such as open source ImageJ. As we show in this study, image processing allows the discrimination among nonaffected cells, reversibly sonoporated cells, and irreversibly sonoporated (dead) cells. Taking all of this into account, our approach represents a simple, reliable, and cheap method to simultaneously evaluate exogenous molecule delivery and cell viability.

The method we present is partially based on a FRET mechanism as proven by fluorescence lifetime measurements. The alternative energy transfer pathway would require photon energy to be released by the donor molecule and reabsorbed by the acceptor, either TB or other intracellular chromophores capable of absorption in donor fluorescence wavelength. Therefore, a proximity-based FRET mechanism makes the combination of EB and TB appropriate for desired quenching and allows the separation among three distinct cell groups. Moreover, EB-TB dye pair could be used as an FRET-based method for the general research in vitro, including multicolor FC applications with tandem fluorophores acting as FRET pairs.

The existence of EB and TB as a specific donor acceptor pair in protein-rich environment (as compared to aqueous environment) clearly identifies the excitation energy loss pathway. Fluorescence quenching effects such as FRET are especially important when the model system (BSA-EtBr-TB) environment changes to the cellular environment. The disadvantage of FRET estimation obtained from the dye-protein interaction in cuvette was that only the average of FRET efficiency over the volume of the sample has been obtained. Usually not all EtBr and TB molecules bind to the protein, and therefore, the calculated FRET efficiency is lowered. Consequently, in cellular environment, the result can be shifted toward higher FRET efficiency due to efficient/preferential EtBr and TB binding to cell DNA and plasma proteins. The investigation of EtBr quenching mechanism in cells would require further investigation including combination of fluorescence lifetime imaging and Förster resonance energy transfer (FLIM-FRET) experimentation.

\section{Disclosures}

No conflicts of interest, financial or otherwise, are declared by the authors.

\section{Acknowledgments}

This work was supported by the grant (MIP-034/2013) from the Research Council of Lithuania.

\section{References}

1. R. Karshafian et al., "Microbubble mediated sonoporation of cells in suspension: clonogenic viability and influence of molecular size on uptake," Ultrasonics 50(7), 691-697 (2010).

2. M. Tamosiūnas et al., "Microbubble sonodestruction rate as a metric to evaluate sonoporation efficiency," J. Ultrasound Med. 31(12), 19932000 (2012).

3. M. Maciulevičius et al., "Analysis of metrics for molecular sonotransfer in vitro," Mol. Pharm. 12(10), 3620-3627 (2015).

4. M. Maciulevičius et al., "Investigation of microbubble cavitationinduced calcein release from cells in vitro," Ultrasound Med. Biol. 42(12), 2990-3000 (2016).

5. J. Park, Z. Fan, and C. X. Deng, "Effects of shear stress cultivation on cell membrane disruption and intracellular calcium concentration in sonoporation of endothelial cells," J. Biomech. 44(1), 164-169 (2011).

6. K. S. Leung et al., "Microbubble-mediated sonoporation amplified lipid peroxidation of Jurkat cells," Chem. Phys. Lipids 180, 53-60 (2014).

7. C. D. Ohl et al., "Sonoporation from jetting cavitation bubbles," Biophys. J. 91(11), 4285-4295 (2006).

8. C. D. Ohl and B. Wolfrum, "Detachment and sonoporation of adherent HeLa-cells by shock wave-induced cavitation," Biochim. Biophys. Acta 1624(1-3), 131-138 (2003).

9. D. L. Miller and C. Dou, "Induction of apoptosis in sonoporation and ultrasonic gene transfer," Ultrasound Med. Biol. 35(1), 144-154 (2009). 
10. N. Lamanauskas et al., "Bleomycin delivery into cancer cells in vitro with ultrasound and SonoVue ${ }^{\circledR}$ or BR $14^{\circledR}$ microbubbles," J. Drug Targeting 21(4), 407-414 (2013).

11. W.-C. Hung, G.-H. Feng, and J.-Y. Cherng, "Fabrication of piezoelectric components for a tunable and efficient device for DNA delivery into mammalian cells," Ultrason. Sonochem. 21(2), 819-825 (2014).

12. D. Baskić et al., "Analysis of cycloheximide-induced apoptosis in human leukocytes: fluorescence microscopy using annexin V/propidium iodide versus acridin orange/ethidium bromide," Cell Biol. Int. 30(11), 924-932 (2006).

13. M. S. Venslauskas, S. Šatkauskas, and R. Rodaitè-Riševičienè, "Efficiency of the delivery of small charged molecules into cells in vitro," Bioelectrochemistry 79(1), 130-135 (2010).

14. B. Jakštys et al., "Different cell viability assays reveal inconsistent results after bleomycin electrotransfer in vitro," J. Membr. Biol. 248(5), 857-863 (2015).

15. I. Ferulova et al. "Photobleaching effects on in vivo skin autofluorescence lifetime," J. Biomed. Opt. 20(5), 051031 (2015).

16. S. R. Sternberg, "Biomedical image processing," Computer 16(1), 22-34 (1983).

17. S. Koch et al., "Ultrasound enhancement of liposome-mediated cell transfection is caused by cavitation effects," Ultrasound Med. Biol. 26(5), 897-903 (2000).

18. P. Qin et al., "Ultrasound-microbubble mediated cavitation of plant cells: effects on morphology and viability," Ultrasound Med. Biol. 38(6), 1085-1096 (2012).

19. S. Phukan and S. Mitra, "Fluorescence behavior of ethidium bromide in homogeneous solvents and in presence of bile acid hosts," J. Photochem. Photobiol. A 244, 9-17 (2012).

20. A. Banerjee et al., "The DNA intercalators ethidium bromide and propidium iodide also bind to core histones," FEBS Open Bio 4(1), 251-259 (2014).

21. W. J. Jin et al., "Fluorescence quenching of ethidium ion by porphyrin cations and quaternary ammonium surfactants in the presence of DNA," Spectrochim. Acta A 53A(14), 2701-2707 (1997).

22. L. M. Davis, J. D. Harvey, and B. C. Baguley, "Electron donor properties of the antitumour drug amsacrine as studied by fluorescence quenching of DNA-bound ethidium," Chem. Biol. Interact. 62(1), 45-58 (1987).

23. S. Bi et al., "Studies of interaction of emodin and DNA in the presence of ethidium bromide by spectroscopic method," Spectrochim. Acta A 69(1), 123-129 (2008).

24. J. Hong et al., "Fluorescence quenching of EB-DNA complex by a novel di-bipyridyl ruthenium(II) complex of p-tert-butyltetrathiacalix [4]arene," Inorg. Chem. Commun. 8(11), 988-991 (2005).

25. P. S. Rabinovitch et al., "BrdU-Hoechst flow cytometry: a unique tool for quantitative cell cycle analysis," Exp. Cell Res. 174(2), 309-318 (1988).
26. M. Poot et al., "Cell-cycle analysis using continuous bromodeoxyuridine labeling and Hoechst 33358: ethidium bromide bivariate flow cytometry," Methods Cell Biol. 41, 327-340 (1994).

27. J. P. Graham et al., "Experimental and theoretical study of the spectral behavior of trypan blue in various solvents," J. Mol. Struct. 1040, 1-8 (2013).

28. G. K. Srivastava et al., "Trypan blue staining method for quenching the autofluorescence of RPE cells for improving protein expression analysis," Exp. Eye Res. 93(6), 956-962 (2011).

29. G. Halldén et al., "Quenching of intracellular autofluorescence in alveolar macrophages permits analysis of fluorochrome labelled surface antigens by flow cytofluorometry," J. Immunol. Methods 142(2), 207-214 (1991).

30. T. Cowen, A. J. Haven, and G. Burnstock, "Pontamine sky blue: a counterstain for background autofluorescence in fluorescence and immunofluorescence histochemistry," Histochemistry 82(3), 205-208 (1985).

31. C. P. Wan, C. S. Park, and B. H. Lau, "A rapid and simple microfluorometric phagocytosis assay," J. Immunol. Methods 162(1), 1-7 (1993).

32. V. Helms, Principles of Computational Cell Biology: From Protein Complexes to Cellular Networks, Wiley-VCH, Weinheim (2008).

33. B. A. Avelar-Freitas et al., "Trypan blue exclusion assay by flow cytometry,” Braz. J. Med. Biol. Res. 47(4), 307-315 (2014).

Paulius Ruzgys received his doctoral degree in biophysics at Vytautas Magnus University in cooperation with Lithuanian University of Health Sciences in 2016. Currently, he works as a researcher in the Biophysical Research Group at Vytautas Magnus University in Kaunas, Lithuania. His main research is focused on sonoporation and electroporation based drug and gene delivery. $\mathrm{He}$ is a member of the European Respiratory Society (ERS), Lithuanian Biophysical Society and Lithuanian Biochemical Society.

Mindaugas Tamošiūnas is a docent in the Department of Biochemistry and a researcher in the Biophysical Research Group at Vytautas Magnus University, Kaunas, Lithuania. He is focused on drug and gene electrotransfer and sonotransfer, laser-tissue interactions, steady state and time-resolved fluorescence spectroscopy, and multispectral imaging as well as on development of implicit dosimetry for sonoporation and photodynamic therapy.

Vanesa Lukinsone (ex. Inesa Ferulova) is a researcher in the Institute of Atomic Physics and Spectroscopy at the University of Latvia. Her main research interests are autofluorescence photobleaching, skin spectral imaging, and fluorescence lifetime.

Saulius Šatkauskas is a professor and head of the Biophysical Research Group at Vytautas Magnus University in Kaunas, Lithuania. His main research is focused on electroporation and sonoporation-based drug and gene delivery. In addition, part of his research work is devoted to developmental and cognitive neuroscience. 